\title{
Adaptation to Conflict via Context-Driven Anticipatory Signals in the Dorsomedial Prefrontal Cortex
}

\author{
Guillermo Horga, ${ }^{1}$ Tiago V. Maia, ${ }^{1}$ Pengwei Wang, ${ }^{1,2}$ Zhishun Wang, ${ }^{1}$ Rachel Marsh, ${ }^{1}$ and Bradley S. Peterson ${ }^{1}$ \\ ${ }^{1}$ Columbia College of Physicians and Surgeons and New York State Psychiatric Institute, New York, New York 10032, ${ }^{2}$ School of Information Science and \\ Engineering, Shandong University, Shandong, People’s Repulic of China 250100
}

Behavioral interference elicited by competing response tendencies adapts to contextual changes. Recent nonhuman primate research suggests a key mnemonic role of distinct prefrontal cells in supporting such context-driven behavioral adjustments by maintaining conflict information across trials, but corresponding prefrontal functions have yet to be probed in humans. Using event-related functional magnetic resonance imaging, we investigated the human neural substrates of contextual adaptations to conflict. We found that a neural system comprising the rostral dorsomedial prefrontal cortex and portions of the dorsolateral prefrontal cortex specifically encodes the history of previously experienced conflict and influences subsequent adaptation to conflict on a trial-by-trial basis. This neural system became active in anticipation of stimulus onsets during preparatory periods and interacted with a second neural system engaged during the processing of conflict. Our findings suggest that a dynamic interaction between a system that represents conflict history and a system that resolves conflict underlies the contextual adaptation to conflict.

\section{Introduction}

The capacity to adjust behavior flexibly is essential for the pursuit of goals in a changing environment. Given sufficient time, humans are able to process environmental stimuli and extract the relevant information needed to guide their choices. For instance, when two differing response tendencies elicited by one stimulus conflict with one another (response conflict), cognitive mechanisms within the prefrontal cortex (PFC) engage to select the most appropriate response (Botvinick et al., 1999; MacDonald et al., 2000; Kerns et al., 2004). Because the environmental context determines the appropriate behavioral response, changing environments compel continuous updating of this cognitive system to produce optimal context-tailored responses (Miller and Cohen, 2001). This contextual adaptability is a central feature of normal cognition (Miller and Cohen, 2001), and its disruption may underlie some neuropsychiatric disorders (Fletcher and Frith, 2009).

Context-driven adaptation is demonstrated in trial-to-trial behavioral adjustments during conflict tasks, such as the Simon task (Simon and Rudell, 1967), even in the absence of explicit predictive cues (Gratton et al., 1992). The context preceding a given stimulus - the sequence of previous trials-prompts adjustments in behavioral responses to the current stimulus. These sequential effects are evident, for example, in the facilitation of

Received June 3, 2011; revised Aug. 11, 2011; accepted Aug. 29, 2011

Author contributions: R.M. and B.S.P. designed research; G.H. performed research; G.H. and P.W. contributed unpublished reagents/analytic tools; G.H., T.V.M., P.W., and Z.W. analyzed data; G.H., T.V.M., R.M., and B.S.P. wrote the paper.

This work was supported in part by NIMH Grants MH089582 and K02-74677, grants from the Alicia Koplowitz Foundation of Spain and the Brain and Behavior Research Foundation (formerly NARSAD), and a Research Associate Award from the New York State Psychiatric Institute and the Research Foundation for Mental Hygiene.

Correspondence should be addressed to Dr. Bradley S. Peterson, 1051 Riverside Drive, Unit 78, New York, NY, 10032.E-mail: PetersoB@childpsych.columbia.edu.

DOI:10.1523/JNEUROSCI.2783-11.2011

Copyright $\odot 2011$ the authors $\quad 0270-6474 / 11 / 3116208-09 \$ 15.00 / 0$ responses to conflict stimuli in sequences wherein conflict predominates (Logan and Zbrodoff, 1979). Sequential adaptation to conflict was originally attributed to a strategic expectancy that relied on a cognitive representation of the task (Gratton et al., 1992), but empirical data supporting this attribution have been lacking thus far (Egner, 2007). To adjust flexibly with context, the neural systems that process and resolve conflict in humans may require updating via a neural system that encodes experienced conflict analogous to a system recently described in nonhuman primates in which activity was dissociable during intertrial intervals (Mansouri et al., 2007). To test this hypothesis in humans, we used a variant of the Simon task to measure context-dependent BOLD responses during non-cued preparatory (intertrial) periods and their relation to behavioral adjustments in 136 healthy participants (aged 7-79 years). Participants were instructed to respond to the direction of arrows (left or right) presented on the left or right side of the screen while ignoring the location of the arrows. Arrow stimuli were congruent (same direction and location) or incongruent (opposite direction and location) in $40 \%$ of the trials each (see Fig. 1). The remaining $20 \%$ of the trials consisted of "blank trials," in which the fixation crosshair that was presented in all intertrial intervals remained on the screen throughout the period during which an active trial would normally be presented.

Based on extant literature, we expected that regions within premotor cortex and anterior cingulate cortex (ACC) would respond to conflict in a context-dependent manner (Botvinick et al., 1999; Carter et al., 2000; Kerns et al., 2004; Nachev et al., 2005). Our main goals were to study whether higher-order brain regions encoded previously experienced conflict during preparatory (intertrial) periods and whether this representation of the context of the task was responsible for trial-to-trial adjustments in behavior. We additionally sought to elucidate the mechanisms 


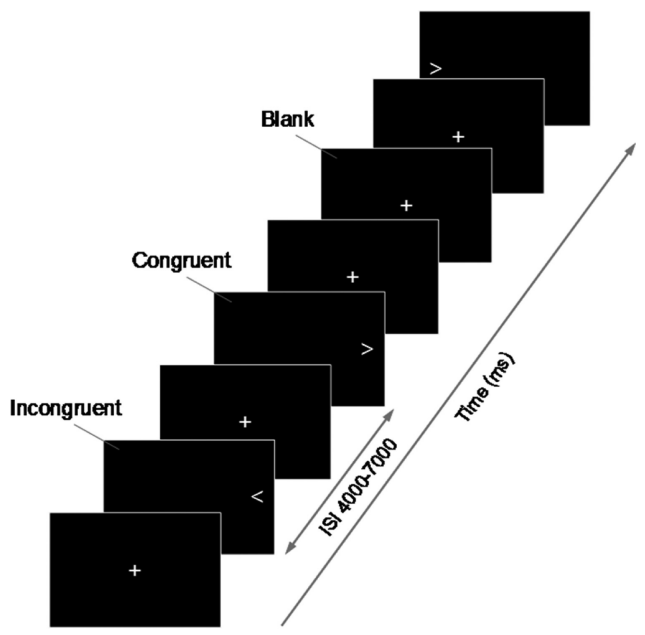

Figure 1. Simon task. Participants responded to the direction of arrow stimuli presented during active (congruent or incongruent) trials. In $20 \%$ of the trials (blank trials), no arrow stimulus was presented (i.e., the fixation crosshair remained on the screen) and no response was required.

that mediate such adjustments by probing brain interactions between consecutive time points in the task.

\section{Materials and Methods}

Subjects

One hundred thirty-six healthy participants participated in the study. (These participants were recruited as controls for our studies of individuals with a variety of psychiatric disorders.) Adult participants provided written informed consent; participants younger than 18 years provided verbal assent, and one of their parents provided written informed consent. The study was conducted in accordance with institutional guidelines (Institutional Review Board of the New York State Psychiatric Institute and the Department of Psychiatry of Columbia University). Participants had no history of neurological illness, past seizures, head trauma with loss of consciousness, mental retardation, pervasive developmental disorder, or any lifetime Axis I psychiatric disorder. Of the 136 participants, 124 (43 younger than 18 years old; 56 females) aged 7-79 years (mean \pm SD age, $24.21 \pm 12.21$ years) with usable imaging and behavioral data were included in the reported analyses.

\section{Experimental design}

Stimuli were delivered through magnet-compatible goggles (Resonance Technologies) using the E-Prime software package. Left- or rightpointing white arrows (subtending $1^{\circ}$ vertical and $4^{\circ}$ horizontal of the visual field) appeared against a black background to either the left or right of the display midline (Fig. 1). Stimuli had $1300 \mathrm{~ms}$ duration and were separated by a jittered interstimulus interval (ISI) of $\sim 4000-7000 \mathrm{~ms}$ (ISI mean \pm SD duration, $5352 \pm 842 \mathrm{~ms}$ ), during which a white gaze fixation crosshair was shown at the midline. The task was divided into three runs. Each run comprised 55 stimuli arranged in pseudorandom order; of these, 22 stimuli were congruent (arrows pointing in the same direction as their position on the screen; 11 rightward-pointing arrows on the right and 11 leftward-pointing arrows on the left), 22 were incongruent (arrows with opposite direction and position; 11 rightwardpointing arrows on the left and 11 leftward-pointing arrows on the right), and 11 were what we refer to as "blank" stimuli, in which the fixation crosshair continued on the screen during the period in which an arrow stimulus would ordinarily have been presented. Participants were instructed to respond as fast and as accurately as possible to the direction of the arrows with a button press on a response box, using the right index finger for a left response and the right middle finger for a right response. Blank stimuli required no response. Responses and reaction times (RTs) were recorded with each button press. A digital interface enabled synchronization of stimulus presentation with scan acquisition within 20 ms. Nearsighted participants were fitted with corrective lenses to ensure that they saw the stimuli clearly.

\section{Behavioral analyses}

We generated a $2 \times 2$ repeated-measures ANOVA with PASW Statistics 18 (SPSS) to test the effects on RT of factors current congruence (F1) (congruent or incongruent) and congruence repetition (F2) (repeated or non-repeated congruence between the preceding and current trial), as well as their interaction. We excluded error trials and trials after error trials, because we were interested in conflict-related and not error-related effects. We also excluded trials with exact stimulus repetitions to control for stimulus-feature priming [the exclusion of exact stimulus repetitions implies that repeated congruence (congruent-congruent trials and incongruent-incongruent trials) always required a response alternation]. In addition to the aforementioned statistical model, we created an alternative model that only differed from the previous one in that F2 represented previous congruence (congruent, incongruent) instead of congruence repetition. To evaluate the influence of the number of consecutive repetitions of congruence (consecutive congruent or incongruent trials) on RTs during the subsequent trial, we used a repeatedmeasures ANOVA with factors current congruence (F1) (congruent, incongruent) and number of preceding consecutive incongruent trials (F2) $(1,2,3,4)$ and a similar, separate ANOVA in which F2 modeled the number of preceding congruent, rather than incongruent, trials. Age was entered as a covariate in all models. We used case-wise exclusion of missing values for all behavioral analyses. (Note that this accounts for slight discrepancies in the degrees of freedom for the various statistical models.) We report Greenhouse-Geisser adjusted values when the sphericity assumption was violated (Mauchly's test, $p<0.05$ ).

\section{Image acquisition}

Functional images were acquired on a 3 T GE Signa EXCITE scanner (GE Medical Systems) with a standard quadrature GE head coil using a singleshot gradient echo planar imaging (EPI) pulse sequence in groups of 34 axial slices per volume and 140 volumes per run ( preceded by six dummy volumes discarded to ensure scanner stability). Head positioning in the scanner was standardized using the canthomeatal line as reference. A T1weighted sagittal localizing scan was used to position the axial images parallel to the anterior commissure-posterior commissure line. T2*-weighted EPI image parameters included the following: $2200 \mathrm{~ms}$ repetition time, $25 \mathrm{~ms}$ echo time, $90^{\circ}$ flip angle, $64 \times 64$ acquisition matrix, $24 \times 24 \mathrm{~cm}$ field of view, $3 \mathrm{~mm}$ slice thickness, $0.5 \mathrm{~mm}$ skip, $62.5 \mathrm{kHz}$ receiver bandwidth, $3.75 \times 3.75 \mathrm{~mm}$ in-plane resolution, interleaved acquisition. Each run lasted $5 \min 8 \mathrm{~s}$, for a total EPI scan time of $15 \min 24 \mathrm{~s}$.

\section{fMRI analyses}

Preprocessing of images was performed using SPM8 (http://www.fil.ion. ucl.ac.uk/spm) and custom Matlab code. We excluded subjects with translation or rotation $>3 \mathrm{~mm}$ or $3^{\circ}$, respectively ( 12 of the initial 136 subjects). EPI volumes were first aligned via least-squares rigid-body transformation, and time series were then despiked from extreme motion and global signal amplitude outliers. Images were slice-timing corrected, resampled to $3 \times 3 \times 3 \mathrm{~mm}^{3}$ voxel size, spatially normalized to an EPI template in Montreal Neurological Institute (MNI) space, and spatially smoothed with a Gaussian kernel of $9 \mathrm{~mm}$ full-width halfmaximum. Finally, time series were high-pass filtered (cutoff frequency of $1 / 128 \mathrm{~Hz}$ via discrete cosine transform) to remove lowfrequency drift.

First-level analyses were performed with SPM8 using a general linear model (GLM) with a weighted least-squares algorithm. Serial correlations in the time series were removed by using a first-order autoregressive model based on a restricted maximum likelihood algorithm. Each stimulus was modeled separately as an independent regressor in the GLM and convolved with a canonical double-gamma hemodynamic response impulse function (Friston et al., 1998). We included the time course of a voxel in the subarachnoid space as a nuisance regressor to account for structured noise in the global signal. Current and preceding trial type (congruent, incongruent, or blank), correct or incorrect response in the current and preceding trial, missing response, and exact stimulus repetition were coded for each event. 
Group analysis of active (i.e., non-blank) trials. The resulting single-trial $\beta$ maps corresponding to correct trials (conditions: 1, congruent-congruent; 2 , incongruent-congruent; 3 , congruent-incongruent; and 4, incongruent-incongruent) without exact stimulus repetitions entered a second-level mixed model on SPM8 ( ${ }_{\mathrm{SPM}}$ ANOVA model). This model consisted of a $2 \times 2$ repeated-measures factorial ANOVA with factors current congruence (F1) (congruent, incongruent) and congruence repetition (F2) (repeated or non-repeated congruence between the preceding and current trial). We assessed main effects of these factors, their interaction, and weighted contrasts by condition. For the latter contrasts, we used the second-order polynomial function fits to the RT-bycondition pattern to generate mean-centered scaled contrasts weighted by the fit to the quadratic polynomial (quadratic-fit-weighted contrast). We applied parametric inference and selected an a priori threshold of uncorrected $p \leq 0.005$. We reported peak-level $p$ values that were familywise error corrected $\left(P_{\mathrm{FWE}}\right)$ according to random field theory.

Group analysis of blank trials. To investigate activation during blank trials and its relation to the preceding active (congruent or incongruent) trials, we created single-trial multilevel multiple regression models using the Multilevel Mediation and Moderation (M3) Toolbox 3.26 (http:// www.columbia.edu/cu/psychology/tor/mediation.html). Blank-trial $\beta$ maps were the dependent variable, and the type of the preceding trial or trials (depending on the specific model used) was the main independent variable. We used nonparametric inference with bootstrap resampling (Wager et al., 2009) with 1000 samples to assess statistical significance (noted as $\left.P_{\text {Bootstrap }}\right)$. We tested three models, each of which used a different independent variable: number of preceding consecutive incongruent trials $\left(\mathrm{M}_{\text {Cumulative }}\right)$, number of preceding consecutive congruent trials $\left(\mathrm{M}_{\text {Cumulative-Congruent }}\right)$, and conflict level in the immediately preceding trial (using quadratic-fit weights by condition; $\mathrm{M}_{\text {Immediate }}$ ). All three models controlled for RT in the preceding trial to avoid carryover effects driven by previous performance. We compared model fits using the Bayesian information criterion (BIC) (Song and Lee, 2006).

Group analysis of the effect of blank trial activation on trial-to-trial RT adjustments. We investigated sequences of active-blank-active trials to assess the effect of activation during blank trials on trial-to-trial RT adjustments. For this purpose, we used an additional single-trial multilevel multiple regression model generated with the M3 toolbox that regressed blank-trial $\beta$ maps $(t-1)$ on trial-to-trial RT changes and their interaction with current congruence (congruent or incongruent at $t$ ). We calculated RT changes as the difference in RTs from the active trial preceding $(t-2)$ to the active trial after $(t)$ the blank trial [RT at $t-\mathrm{RT}$ at $(t-2)]$. We analyzed this RT adjustment effect for incongruent and congruent trials (at $t$ ) separately.

Group analysis of interactions between brain regions in consecutive trials. We defined regions of interest (ROIs) functionally, using the positive findings from the group analyses of active and blank trials. We created ROI masks with the MarsBaR toolbox 0.42 (http://marsbar.sourceforge. net), with each ROI mask corresponding to a positive cluster (thresholded at $p \leq 0.005$, uncorrected). We then extracted and averaged the $\beta$ values from voxels within each ROI mask. To understand the effects that activation during blank trials had on the subsequent trial, we analyzed correlations between ROI activation during blank trials (seed) and whole-brain activation during the subsequent active trial, regardless of stimulus congruence during this active trial. Using the M3 Toolbox, we entered the ROI $\beta$ values by trial (blank trials, $t-1$ ) as the independent variable and the single-trial brain $\beta$ maps of the following trials (the active trial at $t$ after a blank trial) as the dependent variable. This analysis can be considered a variant of a seed-based correlation approach. Next, we focused on the interactions between two ROIs over three consecutive time points (active-blank-active sequences) to understand how activation in these ROIs mutually influenced each other over time to modify responses to current conflict during the second active trial. We dichotomized $\beta$ values in these two ROIs into high and low using a within-subject median split. Finally, we used a reduced-form equations approach to assess the paths between ROIs over three consecutive time points: $t-2, t-1$, and $t$. Three separate repeated-measures ANOVAs with dichotomized activation in the ROIs (high, low) predicted continuous activation in an ROI at a given time point as the dependent variable.
Disentangling the effects of conflict from those attributable to RTs. Conflict is inherently associated with longer RTs, so neural activity associated with conflict trials could stem simply from the longer RTs rather than from conflict per se (Grinband et al., 2011). To address this potential confound, we generated an additional multilevel regression model in which single-trial $\beta$ maps were reorganized into four RT categories independent of conflict level (RT quartile model). For each subject, trials were grouped by RT quartile within each of the four conditions (congruentcongruent, incongruent-congruent, incongruent-incongruent, and congruent-incongruent) and then regrouped into four categories based on the RT quartile, regardless of the condition they pertained (and therefore regardless of the conflict level linked to that condition). By testing the effect of RT-quartile category, this model allowed us to identify brain activation patterns driven by RTs regardless of conflict, which we then compared with the effects of conflict levels.

Time course analyses. To validate further the findings from the image analyses described above, we conducted a model-free analysis of activation time course. This analysis had the advantage of not making any assumptions about the hemodynamic response function. As for all time course analyses for rapid event-related study designs, however, it could not ensure entirely that the BOLD response to one current event was not "contaminated" by the BOLD response to previous events. Nevertheless, the image analyses were not vulnerable to these temporal carryover effects, so that a convergence of findings from both analytic approaches would support their validity. For the time course analyses, we extracted time courses from peak voxels with positive findings, and the surrounding volume within a $10 \mathrm{~mm}$ radius, to create percentage signal change plots time locked to stimulus onset. After partialling out the time course from a voxel in the subarachnoid space and six standard motion parameters, signal from ROIs (collapsed across hemispheres for bilateral positive findings) was bandpass filtered using a zero-phase forward and reverse filter $(0.01<$ frequency $<0.15 \mathrm{~Hz})$ and converted to percentage signal change by first subtracting and then dividing by the stimulus-onset signal amplitude in all time points and then multiplying by 100 . We defined stimulus onset as the baseline for calculation of relative percentage signal change (Konishi et al., 1999; Dolcos et al., 2004; Asplund et al., 2010), which entailed that several of the time course plots show decaying signal from the previous trial in the early portion of the current trial (note that findings from image analyses were not affected by this visual appearance of carryover of signal, because signals from current and previous trials were modeled separately). Time course segments (six to eight repetition times of window length) were then averaged across events and subjects by condition. $p$ values of the obtained group peak-by-condition patterns were computed via a permutation test with 10,000 samples. In the permutation test, conditions were randomly reshuffled within subjects to create each sample. For each of the samples, we extracted subjects' peak amplitude values for each relabeled condition (from the time point corresponding to the peak of the group for the relevant condition) and computed the group average for that condition. Next, we quantified the number of samples meeting a priori criteria that defined the expected pattern (e.g., peak $_{\text {condition1 }}>$ peak condition2 $>$ peak $_{\text {condition3 }}>$ peak $\left._{\text {condition4 } 4}\right)$.

\section{Results}

\section{Contextual adjustment of behavior mirrors BOLD response adaptation}

Analysis of RTs confirmed slower responses to incongruent trials (RT mean, $688.58 \mathrm{~ms}$ ) than to congruent trials (RT mean, 645.91 $\left.\mathrm{ms} ; F_{(1,116)}=5.31, p=0.02\right)$, a difference termed the "interference" or "Simon" effect. Behavioral analysis also demonstrated sequential effects that were independent of exact stimulus repetitions and thus were unlikely to represent stimulus-feature priming. Repeated congruence or incongruence in two consecutive trials (i.e., congruent-congruent or incongruent-incongruent pairs) yielded faster RTs on the second trial of the pair $\left(F_{(1,116)}=6.72, p=0.01\right)$. Furthermore, whether the preceding trial was congruent or incongruent moderated interference measures in the current trial, even after excluding exact stimulus repetitions [Fig. $2 a$; current congru- 

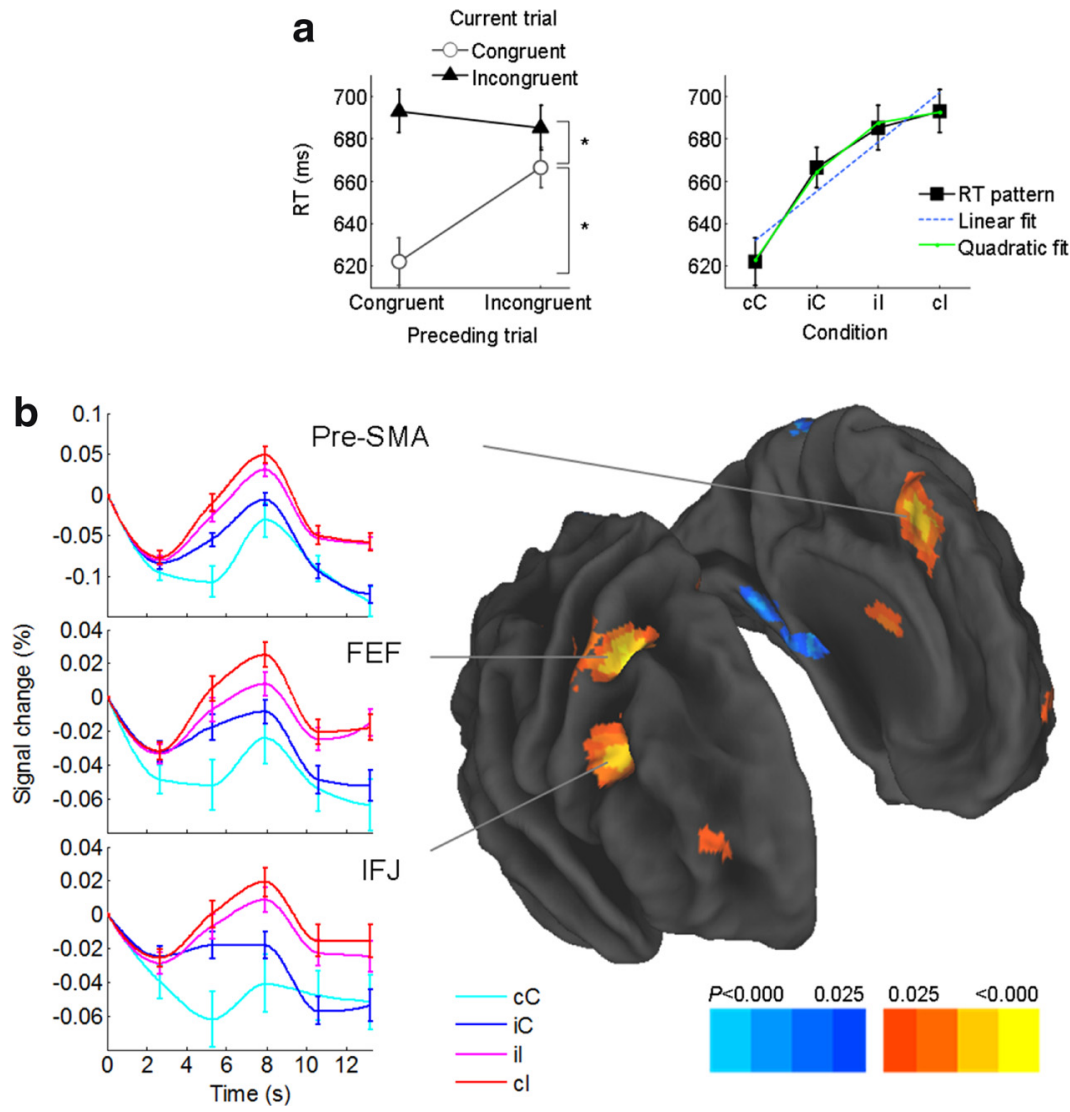

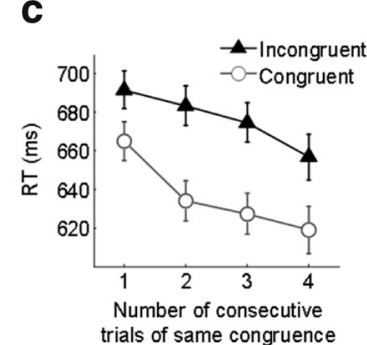

Effect of conflict levels during active triaks

\begin{tabular}{l|cccc}
\hline & $\begin{array}{c}\text { MNI } \\
\text { Reordinates } \\
(x, y, z)\end{array}$ & $k_{E}$ & $z$ & $P_{\mathrm{FWE}}$ \\
\hline Left pre-SMA & $6,-1,55$ & 227 & 4.06 & 0.008 \\
& $-9,-7,52$ & & 3.70 & 0.029 \\
Right FEF & $33,-10,49$ & 107 & 3.78 & 0.022 \\
Right IFJ & $42,8,28$ & 32 & 3.51 & 0.054 \\
Right precuneus & $6,-64,64$ & 73 & -4.51 & 0.003 \\
& $12,-61,70$ & & -4.16 & 0.014 \\
Right PG-LG & $9,-52,1$ & 152 & -3.98 & 0.029 \\
Left PG-LG & $-9,-58,-2$ & & -3.84 & 0.049 \\
\hline
\end{tabular}

Figure 2. Effects of conflict during active trials. $\boldsymbol{a}$, Left, Mean RTs for congruent and incongruent stimuli depending on the preceding congruence. All pairwise post hoc Sidak's tests (* $p<0.01)$ except for incongruent-incongruent versus incongruent- congruent $(p=0.6)$. Right, Mean RTs for conditions ranked from fastest to slowest RTs ( $c$ C, congruent- congruent; iC, incongruent- congruent; il, incongruentincongruent; $\mathrm{cl}$, incongruent- congruent) and linear and quadraticfits to the RT-by-condition pattern (both $p$ values $<0.0001$ ). $\boldsymbol{b}$, Middle, Brain regions with increasing (hot colors) or decreasing (blue) response to increasing levels of conflict during active trials (quadratic-fit-weighted contrast, sPMANOVA) and the corresponding peak-level statistics (right; search volume confined to areas with task effects; $k_{\mathrm{E}}$ cluster extent in adjacent voxels; $\mathrm{PG}-\mathrm{LG}$, parahippocampal-lingual gyri). Surface renderings generated with Caret software. Left, Time course plots depicting percentage BOLD signal change by condition from stimulus presentation (at time 0 ) in the FEFs, IFJ, and pre-SMA. Note that the decay in activity observed during the first $2 \mathrm{~s}$, which might reflect carryover signal from the previous trial, does not affect the imaging analysis in $\boldsymbol{b}$, in which the current and previous trial are modeled separately (see Materials and Methods). In addition, this potential carryover signal does not appear to depend on the previous trial type, so it is unlikely to confound our analyses or affect our conclusions. $c$, RTs during active trials show a monotonic decline as a function of the number of preceding consecutive trials of the same congruence type (linear contrasts: $F_{(1,119)}=5.8, p=0.018$, and $F_{(1,122)}=11.36, p<0.001$, for incongruent and congruent trials, respectively). Error bars indicate SEM.

ence $\times$ preceding congruence (congruent, incongruent) interaction, $\left.F_{(1,116)}=6.72, p=0.01\right]$. This sequential moderation of interference by the presence or absence of previous conflict was consistent over time (three-way interaction with run, $p=0.85$ ). Interference (incongruent - congruent RT mean) was appreciably greater after congruent $(68.65 \mathrm{~ms})$ than after incongruent trials $\left(16.68 \mathrm{~ms} ; F_{(1,116)}=\right.$ $6.72, p=0.01$ ), which we term the "interference adaptation effect." This effect was driven primarily by sequential effects affecting current congruent trials (incongruent-congruent vs congruent-congruent trials, $p<0.0001$, post hoc Sidak's test; Fig. 2a). RTs increased in a curvilinear manner from the slowest to the fastest of four conditions defined by congruence of the current and preceding trial (congruent-congruent, incongruent-congruent, incongruent-incongruent, and congruent-incongruent trials; Fig. 2a), suggesting the presence of various levels of conflict that depend on the congruence of the current and preceding trials. None of these effects varied with age (all $p$ values $>0.1$ ).

We used a parametric approach to identify voxels in which activity mirrored this behavioral pattern across conditions, thereby comprising context-adaptive brain regions likely driving this pattern. We modeled trial-by-trial BOLD signal changes and derived weighted contrasts from the second-order polynomial fit to the RTby-condition behavioral pattern (Fig. 2a; see Materials and Meth- ods). A cluster of voxels extending on the left hemisphere from the pre-supplementary motor area (pre-SMA) to the dorsal ACC (dACC) parametrically increased in activation with increasing levels of conflict (Fig. 2b). A similar graded response pattern was identified in a portion of the right precentral gyrus overlapping with the frontal eye fields (FEFs) and in the right inferior frontal junction (IFJ; Fig. $2 b$; quadratic-fit-weighted contrast: all peaks $t_{(486)}>2.9, P_{\mathrm{FWE}}<$ 0.05 , search extent confined to task-positive regions). All three areas evidenced a current-congruence $X$ preceding-congruence interaction (all peaks $t_{(486)}>3.4, p<0.0001$, uncorrected; $P_{\mathrm{FWE}}<0.05$, search extent confined to areas with graded response), closely resembling the observed context-driven adjustments in behavior (Fig. $2 b$ ). The bilateral parahippocampal gyri and right precuneus followed an inverse pattern, exhibiting more deactivation in conditions with more conflict. Within the areas with a graded BOLD response to varying levels of conflict, a conjunction analysis (Friston et al., 1999) indicated that the pre-SMA and IFJ activated more during trials in which congruence alternated relative to the previous trial than during trials in which congruence types repeated [i.e., (congruent-incongruent vs congruent-congruent) $\cap$ (incongruent-congruent vs incongruent-incongruent) trials; $t_{(486)}>3.4, p<0.0001$, uncorrected; $\left.P_{\mathrm{FWE}} \leq 0.05\right]$. Pre-SMA activation was also greater for alternation than for repetition trials within current trials that were 
congruent (incongruent-congruent vs congruent-congruent trials; $t_{(486)}=3.57, p<$ 0.0001 , uncorrected; $P_{\text {FWE }}=0.039$ ), indicating that the pre-SMA was also engaged in the absence of current-trial conflict. Once we accounted for the sequential effects of congruence, we were unable to dissociate patterns of activation that might have represented distinct mechanisms of cognitive control for incongruent-congruent versus incongruent-incongruent trials. In contrast with the view that stimulus-feature priming explains adaptation to conflict (Mayr et al., 2003), we found marked adaptation of behavior and BOLD response when excluding exact stimulus repetitions (as in all analyses reported above). For subsequent analyses, we focused on the pre-SMA region-ofinterest (pre-SMA $\mathrm{ROI}_{\mathrm{I}}$, defined functionally) because of its documented role in the resolution of conflict (Nachev et al., 2005; Isoda and Hikosaka, 2007).

\section{Sequencing of trial congruence conditions prepotency}

Although stimulus presentation was pseudorandom, the consecutive recurrence of trials of the same congruence type (i.e., consecutive congruent or consecutive incongruent trials) cumulatively facilitated RTs (Fig. 2c). Demonstrating an adaptation to conflictladen stimuli, incongruent-trial repetitions produced monotonic declines in RTs and in peak amplitude of pre-SMA $\mathrm{ROI}_{\mathrm{R}}$ hemodynamic responses (linear contrast, $F_{(1,120)}=4.42, p=0.04$ ). Furthermore, incongruent-trial repetitions had opposite effects on subsequent incongruent and congruent trials [current-congruence $X$ number of preceding consecutive incongruent trials $(\leq 2$, $>2$ ) interaction, $F_{(1,119)}=5.54, p=0.02$ ], such that, after more than two consecutive incongruent trials, the interference effect reversed (i.e., RTs became longer for congruent than for incongruent trials by $34.09 \mathrm{~ms}$ ), as did the pattern of neural responses in the pre-SMA (although nonsignificantly). Such contextrelated reversal of interference strongly suggests that contextual patterns condition prepotency over time.

\section{Previous conflict drives preparatory BOLD responses}

To elucidate the mechanism of trial-to-trial adjustments in the response to conflict, we targeted the periods during which such adjustments are likely to occur: the intertrial intervals (Mansouri et al., 2007). Our design was especially suitable for assessing context-driven preparatory activation during intertrial intervals as a result of the inclusion of blank trials, inserted occasionally when an active (congruent or incongruent) trial would be expected. Blank trials-essentially, long intertrial intervals-provided a sufficient time window for exploring preparatory changes in BOLD signal related to stimulus anticipation. In contrast with previous studies probing cue-induced preparatory activation (Barber and Carter, 2005; Luks et al., 2007; Sohn et al., 2007; Aarts et al., 2008), we investigated the influence of the preceding context on activation during non-cued blank trials. Conflict in the preceding trial was associated with greater activation during blank trials in the left dorsomedial PFC (DMPFC), left angular gyrus, and left precuneus (incongruent-blank vs congruent-blank; Fig. 3; see Materials and Methods), areas that deactivated in response to active trials. Although an oddball effect could explain activation during blank trials, in which expected stimuli were not presented, contrasting two equally surprising conditions (incongruent-blank vs congruent-blank) precluded this interpretation. Moreover, preparatory activation, especially in the DMPFC, evidenced a dose-response pattern that was proportional to the number of previous consecutive incongruent trials, regardless of RTs on the preceding trial (all peaks $P_{\text {Bootstrap }}<0.001$; Fig. 4a,b; see Materials and Methods). This cumulative conflict model provided a better fit than an alternative model that regressed blank trial activation against conflict only during the immediately preceding trial $\left(\mathrm{M}_{\text {Immediate }}\right.$; BIC based on $\mathrm{DMPFC}_{\mathrm{ROI}}$ error variance: $\mathrm{BIC}_{\text {Immediate }}=114$ and $\mathrm{BIC}_{\text {Cumulative }}=53$, in support of $\mathrm{M}_{\text {Cumulative }}$ ). Additionally, a model-free analysis of DMPFC time courses confirmed a monotonic increase in the magnitude of BOLD signal peaks with an increasing number of previous consecutive incongruent trials (Fig. $4 b$; see Materials and Methods) but not as a direct function of the conflict level in the immediately preceding trial. The same regions that exhibited increasing activity with increasing number of consecutive incongruent trials exhibited decreasing activity with increasing number of consecutive congruent trials (Fig. 4c), suggesting that activity in these regions specifically encoded conflict history. Because stimulus presentation was pseudorandom, participants were unable to guess the upcoming stimulus congruence given the preceding sequence. Preparatory activation was thus unlikely to reflect an estimate of the objective probability of future stimulus congruence. Instead, preparatory activation was biased by the contextual pattern of past conflict trials (i.e., conflict history). We focused on the $\mathrm{DMPFC}_{\mathrm{ROI}}$, a region implicated in response preparation (Haynes et al., 2007; Soon et al., 2008), to determine how its encoding of conflict history influenced subsequent responses.

\section{Preparatory BOLD responses mediate trial-to-trial adaptations to conflict}

We assessed the behavioral effects of preparatory activation by testing the influence of activation during blank trials $(t-1)$ on trial-to-trial adjustment of RTs on the subsequent (active) trial $(t)$. If encoding conflict history in the DMPFC confers a behavioral advantage, preparatory activation in this region should drive the observed RT facilitation in conflict (incongruent) trials 


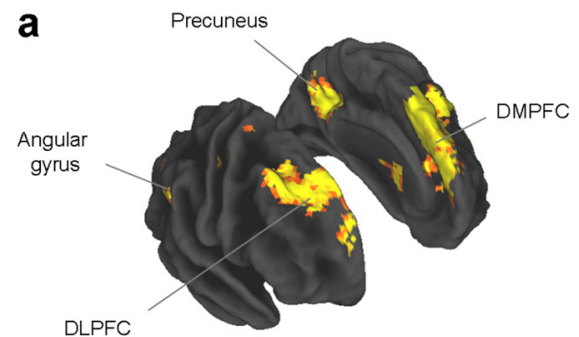

b

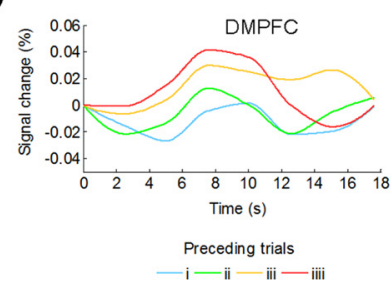

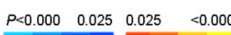

C

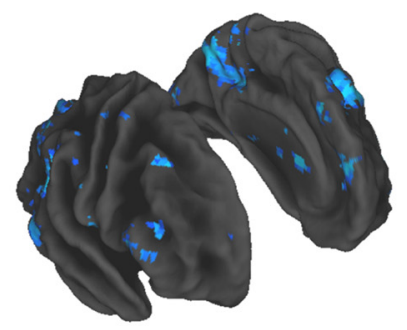

e

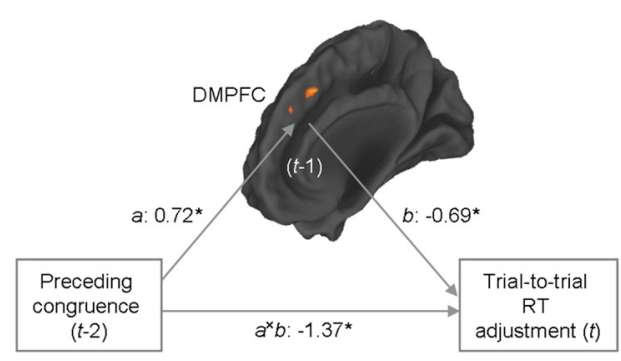

d
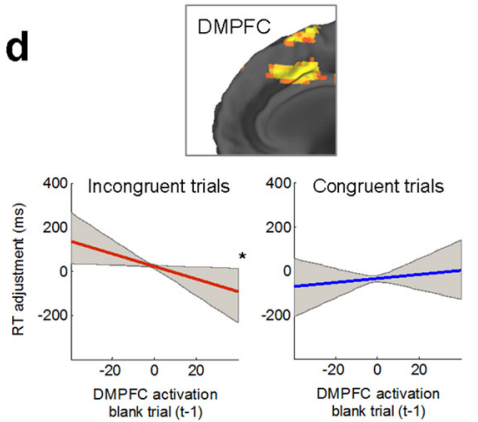

f

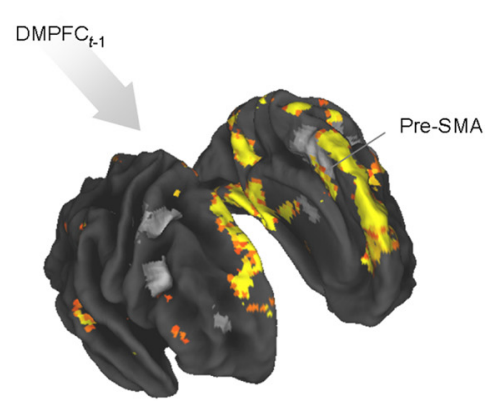

Figure 4. Effects of cumulative conflict on subsequent preparatory activation during blank trials. $\boldsymbol{a}$, Brain regions in which increasing number of preceding consecutive incongruent trials correlates with increasing activation during the subsequent blank trial. Note that the positive cluster centered in the DMPFC connects with part of the dorsolateral prefrontal cortex (DLPFC) within Brodmann area 9. $\boldsymbol{b}$, Time course plots of DMPFC [MNI coordinates (mm): $\pm 9,55,24]$ activation for blank trials preceded by $1,2,3$, or 4 consecutive incongruent trials (pattern $p=0.04$, permutation test). $c$ Effects of preceding consecutive congruent trials. $\boldsymbol{d}$, Top, Brain regions in which activation during blank trials predicts greater RT adjustment for subsequent incongruent trials more than for subsequent congruent trials (RT adjustment $\times$ current congruence at $t$ ). Bottom, Group regression slopes (group average in color; $95 \%$ confidence interval in gray) for the relationship between activation in the $\mathrm{DMPFC}_{\mathrm{ROI}}(t-1)$ and trial-to-trial RT adjustments on the subsequent trial $(t)$ by congruence. $\boldsymbol{e}$, Mediation model. Standardized path coefficients for the peak voxel [MNI coordinates (mm): $-6,41,25]$ are presented for $a$ and $b$ paths. The negative effect of path $b$ represents that activation in the DMPFC correlates with acceleration of RTs on subsequent incongruent trials. The $a \times b$ effect (or mediation effect) represents a significant reduction of the direct path effect (that is, the path between congruence in the preceding trial and RT adjustment) when controlling for DMPFC activation during blank trials (bootstrap test). The brain map depicts voxels with significant mediation $(a \times b)$ effects. ${ }^{*} p \leq 0.05$. $\boldsymbol{f}$, Brain regions in which $\mathrm{DMPFC}_{\mathrm{ROI}}$ activation in the preceding blank trial $(t-1, a)$ determines BOLD response at time $t$ (active trial after blank trial; whole-brain seed correlation). Regions colored in light gray had significant effects of conflict level (see Fig. 1b). Maps are thresholded at $P_{\text {Bootstrap }}<0.025$.

that follow other conflict trials (Fig. 2c). This hypothesized effect was confirmed, in that greater activation within the right DMPFC during blank trials $(t-1)$ predicted greater acceleration of RTs on the subsequent trial (i.e., RTs at $t$ speeded up to a greater extent relative to $t-2$ ) for incongruent, but not for congruent, trials (Fig. $4 d$; RT adjustment $\times$ current congruence, $\left.P_{\text {Bootstrap }}<0.005\right)$. Indeed, a mediation analysis supported that preparatory activation within the DMPFC mediated the effects of preceding conflict on RT adjustment in the subsequent incongruent trial (Fig. $4 e$; see Materials and Methods).

Having found that preparatory DMPFC activation encoded the history of preceding conflict (Fig. $3 a-c$ ) and that preceding con-

flict modulated neural response to current conflict in the pre-SMA and other areas, mirroring behavior (Fig. 2a,b), we next assessed seed-based functional connectivity to test our prediction that preparatory DMPFC activation (seed ROI at $t-1$ ) produces subsequent context-driven adaptation in the pre-SMA $(t)$, consistent with previously documented direct projections from DMPFC to rostral supplementary motor and cingulate cortices (Petrides and Pandya, 2007). We used a $\beta$ series correlation approach (Rissman et al., 2004) for this analysis because it is suitable for studying stage- or period-specific brain interactions such as those we hypothesized (i.e., interactions of preparatory periods when a stimulus was expected with activation after actual stimulus presentation). As we predicted, preparatory activation in the $\mathrm{DMPFC}_{\mathrm{ROI}}$ $(t-1)$ correlated positively with subsequent activation $(t)$ in the pre-SMA $A_{\mathrm{ROI}}$, even after partialling out shared variance with another task-positive area (partial correlation controlling for $\mathrm{IFJ}_{t}, r=0.11, p<$ 0.0001 ) and also enhanced subsequent activation in visual and parahippocampal areas (Fig. 4f). Path analysis of factors contributing to pre-SMA activation at $t$ revealed a complex modulation pattern (Fig. $5 a$; see Materials and Methods): preparatory DMPFC $_{\text {ROI }}$ activation $(t-1)$ modulated prestimulus activation in the pre-SMA $\mathrm{ROI}_{\text {, }}$ and this prestimulus activation conditioned different responses to current congruence in the pre-SMA $\mathrm{ROI}_{\mathrm{I}}$ during stimulus presentation $(t)$, suggesting that the DMPFC indirectly modulated pre-SMA response to conflict. Moreover, preceding activity in the $\mathrm{DMPFC}_{\mathrm{ROI}}$ modulated the neural response to current conflict in the pre-SMA $\mathrm{ROI}_{\text {in the }}$ same way that preceding conflict modulated the behavioral response to current conflict (Fig. 5b,c compared with Fig. 2a). Preparatory activity in both the precuneus and angular gyrus, regions that tightly coactivated with the DMPFC during blank trials, also appeared to modulate response to conflict in the pre-SMA ${ }_{\mathrm{ROI}}$ (and therefore satisfied criteria $c_{1}$ and $c_{2}$ but not $c_{3}$; listed in the legend for Fig. $5 b$ ) but not in other taskpositive regions. As expected for a region implicated in conflict processing, response amplitude in the pre-SMA $\mathrm{ROI}_{\text {at }}$ acorrelated positively with RTs $\left(F_{(1,116)}=57.38, p<0.0001\right)$ but was not simply explained by RTs, because its activation was uncorrelated with RTs when controlling for conflict level ( $p=0.45$; Fig. 6 ; see Materials and Methods). Last, we found additional support for the role of preparatory DMPFC activation in behavioral adaptation. Participants who adapted better to interference had greater preparatory activation in the $\mathrm{DMPFC}_{\mathrm{ROI}}$ than those who adapted less well (abovemedian vs below-median interference adaptation effect; $t_{114}=1.73$, $p=0.04$, one-tailed). 


\section{Discussion}

Our findings indicate that the pre-SMA and other prefrontal areas have a graded response to conflict that closely mirrors behavioral performance on a conflict task. Previously experienced conflict moderated both hemodynamic and behavioral responses to conflict. Electrophysiological data from monkeys suggest that the pre-SMA detects and resolves conflict by switching from automatic to controlled goal-oriented actions after contextual cues (Isoda and Hikosaka, 2007). Humans might likewise resolve conflict by switching proactively from prepotent (automatic) to controlled (less automatic) response modes in a contextdependent manner. The main effect of current stimulus congruence reflects an inherent response conflict that appears when goal-oriented response tendencies compete with a prepotency established early in life (i.e., an inherent prepotency). The preceding context establishes a second type of prepotency, one that is based on recently experienced context and that facilitates behavior on sequences of similar stimuli (wherein similarity may be at a feature level, as in simple forms of priming, or at a more abstract level, as in our present investigation, in which similarity refers to whether consecutive stimuli are congruent or incongruent). Conversely, this contextual prepotency constitutes a source of context-related response conflict that impairs performance on sequences of dissimilar stimuli. Contextual response conflict was most evident in the reversal of interference on stimuli that followed sequences of two or three incongruent stimuli. A common neural pathway to solve both inherent and contextual response conflict may involve a switch to controlled response modes. Supporting this hypothesized common conflict-solving stream, our results indicate that pre-SMA and related structures engage when goal-oriented response tendencies compete with inherent or contextually favored prepotency, consistent with previous reports of pre-SMA-dACC activation in response to violations of context-based predictions (Huettel et al., 2002) and the prediction error signals found in neurons within the macaque homolog of the human ACC (Matsumoto et al., 2007). Furthermore, the statistical interaction of current and preceding congruence detected in these prefrontal areas suggests that these regions undergo adjustments in the inherent response conflict based on the preceding context.

We sought to understand the mechanisms of this contextual adjustment of responses to conflict and whether a general stimulus-type priming or conflict-specific information triggered this adjustment. A system comprising the DMPFC, precuneus, and inferior parietal cortex specifically encoded a representation of conflict history across successive trials and influenced the neural and behavioral adaptation to conflict. This system deactivated during active trials but activated in a dose-response manner to the number of preceding conflict-laden (incongruent) trials during intertrial intervals (blank trials), when stimulus onsets were antici-

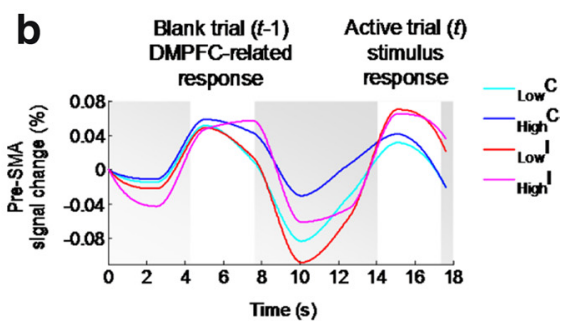

c

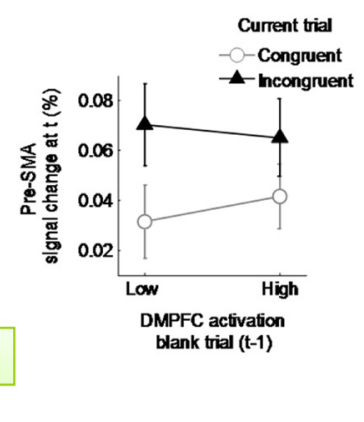

$\mathrm{RT}$ at $(t)$

during subsequent active trials. $\boldsymbol{a}$, Path diagram depicting relationships between DMPFC and pre-SMA at three consecutive time points (active-blank-active sequences). Three the subsequent active trial, respectively), confirming modulation by incongruent) at $t\left(c_{1}\right)$ with opposite effect depending on current congruence $\left(c_{2}\right)$ and greater effect on congruent trials than incongruent trials $\left(c_{3}\right)$ (pattern $p=0.001$, meeting $c_{1}-c_{3}$ criteria, permutation test). $c$, Pre-SMA hemodynamic response peaks by DMPFC activation at $t-1$. Error bars represent SEM.
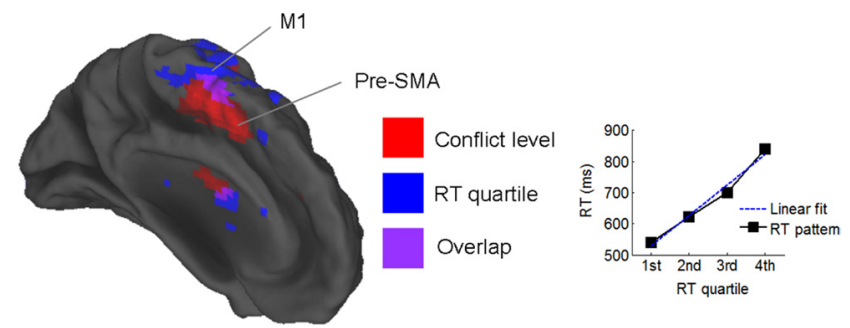

Figure 6. RT quartile model. Left, Comparison of neural response to conflict levels

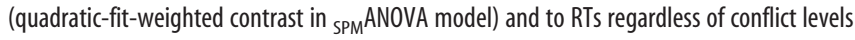
(main effect of quartile in RT quartile model; see Materials and Methods). Only a minimum overlap between the conflict-related and the purely RT-related regions is noticeable. Both contrasts are thresholded at $P_{\text {Bootstrap }}<0.025$. M1, Primary motor cortex. Right, Mean RTs and SEM by RT quartile and linear fit to the RT-by-RT quartile pattern.

pated. The regions sensitive to conflict history overlapped with a "task-negative" network consistently implicated in episodic memory and prospective thinking (Addis et al., 2007). The rostral DMPFC, a constituent of this network, has been found to participate selectively in prospective memory (Reynolds et al., 2009), which involves remembering to perform a goal-oriented action at the appropriate moment and to encode task-specific goals during response preparation (Haynes et al., 2007). Lesion studies further substantiate the role of the rostral DMPFC in prospective memory (Burgess et al., 2000) and in the adaptation to conflict (di Pellegrino et al., 2007). Task-negative regions, such as the DMPFC and precuneus, might encode goal-relevant information during response preparation and influence subsequent response selection in task-positive regions, 
including the pre-SMA and SMA (Soon et al., 2008). Tasknegative and task-positive regions appear as temporally anticorrelated systems (Greicius et al., 2003), yet they may interact to adjust performance of controlled processes (Northoff et al., 2010). Indeed, our analyses demonstrated that a task-negative region, the DMPFC, modulates task-related activity during subsequent trials at both a neural and behavioral level. Our data further suggest a potential mechanism underlying this modulation: the rostromedial PFC, typically recruited by higher-order processes (Sakai and Passingham, 2003; Forstmann et al., 2006; Haynes et al., 2007; Coricelli and Nagel, 2009), amplifies prestimulus neural activity at the conflict-processing site in the preSMA in a top-down manner, thereby enhancing task-relevant processing pathways and facilitating response selection in accordance with contextual demands (Botvinick et al., 2001). This top-down modulation may represent an instance of the anticipatory biasing that has been hypothesized to occur at preparatory periods of controlled processing and that has been demonstrated during the encoding period of a selective-attention task (Zanto et al., 2011).

Our findings are human analogs of findings from single-cell recordings in monkeys, in which principal sulcus cells responded to previous conflict during the intervals between active trials (Mansouri et al., 2007). Memory of conflict in this cell population was deemed key for context-driven adaptations to conflict. In line with this and previous accounts (Gratton et al., 1992), our findings in humans suggest that a representation of conflict history encoded in the DMPFC (and portions of the dorsolateral PFC) drives context-related adjustments in the response to current conflict. Rather than representing a memory trace per se, activity in the DMPFC could alternatively represent either an updating operation of strategy or the strengthening of goal maintenance in conflict-rich contexts that render inherent response tendencies suboptimal (Miller and Cohen, 2001; Bunge et al., 2003; Hampton et al., 2008). Regardless of the cognitive process ascribed to the preparatory activation we identified in the DMPFC, our findings underscore the importance of activity during the preparatory period in supporting trial-to-trial behavioral adjustments to conflict (Mansouri et al., 2009), consistent with reports of anticipatory activity associated with cued adjustments to conflict (Sohn et al., 2007; Aarts et al., 2008).

In general, our imaging and behavioral findings are compatible with a previously proposed computational model for conflict monitoring (Botvinick et al., 2001). That model, however, does not account for certain of our observations, particularly the context-dependent effects on current congruent trials and the reversal of interference, effects that have also been observed by others (Stürmer et al., 2002). In contrast, an expectancy account of adaptation to conflict would posit that high implicit or explicit expectancy for an incongruent trial induces contextual conflict when an unexpected congruent trial is presented, thereby impairing performance, as we observed. A recent extension to the original conflict-monitoring model (Botvinick, 2007), however, can also in principle accommodate both context-dependent effects on current congruent trials and the reversal of interference that we observed. This extended model holds that conflict during the application of a particular strategy serves as a teaching signal that influences subsequent strategy selection. The preference for a given strategy is reinforced in a specific context (e.g., a particular strategy that is advantageous for responding to incongruent stimuli but disadvantageous for congruent stimuli could become the preferred strategy in the context of an incongruent-rich sequence), and this strategy preference could therefore induce a disruption in performance after contextual changes (e.g., during a congruent trial that follows a train of consecutive incongruent trials).

The extent to which the optimal strategy for incongruent trials disrupts performance in congruent trials can vary across tasks, which could account for the differences in sequential effects on behavior that have been reported across differing conflict tasks. Studies using the Simon task, for example, have reported that the congruence of the previous trial strongly influences the RT of a current congruent trial (Kerns, 2006; Fischer et al., 2008), whereas studies using the Stroop task have reported this influence to be almost negligible (Kerns et al., 2004). This difference in the effects of temporal context of conflict on performance could mean that the appropriate strategy used during incongruent trials carries the disadvantage of disrupting performance on subsequent congruent trials in the Simon task but that the same disadvantage does not occur (or at least it occurs to a lesser extent) in the Stroop task. Indeed, a reasonable strategy in a context that is highly enriched with incongruent trials in the Simon task would be simply to respond to the side opposite the stimulus, a strategy that would strongly impair performance during congruent trials. A similar strategy does not seem to be available in the Stroop task, at least when more than two color responses are possible (as in the study of Kerns et al., 2004), in which case any optimal strategy for response to incongruent trials in the Stroop task would have a less detrimental effect on performance during congruent trials. Despite these relatively minor differences in behavioral effects that differing response strategies during conflict trials have across differing conflict tasks, our findings overall emphasize the role of contextual patterns in the ongoing adaptation to conflict and support computational models that account for these higherorder sequential effects (Cho et al., 2002; Bunge et al., 2003; Brown et al., 2007).

We have presented evidence that human adaptation to conflict relies on the interaction of two dissociable neural systems: first, a stimulus-responsive system that activates with current conflict in a context-dependent manner and, second, a preparatory system that encodes contextual information related to previously experienced conflict. By continually incorporating contextual information into the ongoing process of response selection, the dynamic interaction of these two systems may tune behavior to the rapidly changing experiential demands of everyday life.

\section{References}

Aarts E, Roelofs A, van Turennout M (2008) Anticipatory activity in anterior cingulate cortex can be independent of conflict and error likelihood. J Neurosci 28:4671-4678.

Addis DR, Wong AT, Schacter DL (2007) Remembering the past and imagining the future: common and distinct neural substrates during event construction and elaboration. Neuropsychologia 45:1363-1377.

Asplund CL, Todd JJ, Snyder AP, Marois R (2010) A central role for the lateral prefrontal cortex in goal-directed and stimulus-driven attention. Nat Neurosci 13:507-512.

Barber AD, Carter CS (2005) Cognitive control involved in overcoming prepotent response tendencies and switching between tasks. Cereb Cortex 15:899-912.

Botvinick MM (2007) Conflict monitoring and decision making: reconciling two perspectives on anterior cingulate function. Cogn Affect Behav Neurosci 7:356-366.

Botvinick MM, Braver TS, Barch DM, Carter CS, Cohen JD (2001) Conflict monitoring and cognitive control. Psychol Rev 108:624-652.

Botvinick M, Nystrom LE, Fissell K, Carter CS, Cohen JD (1999) Conflict monitoring versus selection-for-action in anterior cingulate cortex. $\mathrm{Na}$ ture 402:179-181.

Brown JW, Reynolds JR, Braver TS (2007) A computational model of frac- 
tionated conflict-control mechanisms in task-switching. Cogn Psychol 55:37-85.

Bunge SA, Kahn I, Wallis JD, Miller EK, Wagner AD (2003) Neural circuits subserving the retrieval and maintenance of abstract rules. J Neurophysiol 90:3419-3428.

Burgess PW, Veitch E, de Lacy Costello A, Shallice T (2000) The cognitive and neuroanatomical correlates of multitasking. Neuropsychologia 38:848-863.

Carter CS, Macdonald AM, Botvinick M, Ross LL, Stenger VA, Noll D, Cohen JD (2000) Parsing executive processes: strategic vs. evaluative functions of the anterior cingulate cortex. Proc Natl Acad Sci U S A 97:1944-1948.

Cho RY, Nystrom LE, Brown ET, Jones AD, Braver TS, Holmes PJ, Cohen JD (2002) Mechanisms underlying dependencies of performance on stimulus history in a two-alternative forced-choice task. Cogn Affect Behav Neurosci 2:283-299.

Coricelli G, Nagel R (2009) Neural correlates of depth of strategic reasoning in medial prefrontal cortex. Proc Natl Acad Sci U S A 106:9163-9168.

di Pellegrino G, Ciaramelli E, Làdavas E (2007) The regulation of cognitive control following rostral anterior cingulate cortex lesion in humans. J Cogn Neurosci 19:275-286.

Dolcos F, LaBar KS, Cabeza R (2004) Interaction between the amygdala and the medial temporal lobe memory system predicts better memory for emotional events. Neuron 42:855-863.

Egner T (2007) Congruency sequence effects and cognitive control. Cogn Affect Behav Neurosci 7:380-390.

Fischer R, Dreisbach G, Goschke T (2008) Context-sensitive adjustments of cognitive control: conflict-adaptation effects are modulated by processing demands of the ongoing task. J Exp Psychol Learn Mem Cogn 34:712-718.

Fletcher PC, Frith CD (2009) Perceiving is believing: a Bayesian approach to explaining the positive symptoms of schizophrenia. Nat Rev Neurosci 10:48-58.

Forstmann BU, Brass M, Koch I, von Cramon DY (2006) Voluntary selection of task sets revealed by functional magnetic resonance imaging. J Cogn Neurosci 18:388-398.

Friston KJ, Josephs O, Rees G, Turner R (1998) Nonlinear event-related responses in fMRI. Magn Reson Med 39:41-52.

Friston KJ, Holmes AP, Price CJ, Büchel C, Worsley KJ (1999) Multisubject fMRI studies and conjunction analyses. Neuroimage 10:385-396.

Gratton G, Coles MG, Donchin E (1992) Optimizing the use of information: strategic control of activation of responses. J Exp Psychol Gen 121:480-506.

Greicius MD, Krasnow B, Reiss AL, Menon V (2003) Functional connectivity in the resting brain: a network analysis of the default mode hypothesis. Proc Natl Acad Sci U S A 100:253-258.

Grinband J, Savitskaya J, Wager TD, Teichert T, Ferrera VP, Hirsch J (2011) The dorsal medial frontal cortex is sensitive to time on task, not response conflict or error likelihood. Neuroimage 57:303-311.

Hampton AN, Bossaerts P, O'Doherty JP (2008) Neural correlates of mentalizing-related computations during strategic interactions in humans. Proc Natl Acad Sci U S A 105:6741-6746.

Haynes JD, Sakai K, Rees G, Gilbert S, Frith C, Passingham RE (2007) Reading hidden intentions in the human brain. Curr Biol 17:323-328.

Huettel SA, Mack PB, McCarthy G (2002) Perceiving patterns in random series: dynamic processing of sequence in prefrontal cortex. Nat Neurosci 5:485-490.

Isoda M, Hikosaka O (2007) Switching from automatic to controlled action by monkey medial frontal cortex. Nat Neurosci 10:240-248.

Kerns JG (2006) Anterior cingulate and prefrontal cortex activity in an FMRI study of trial-to-trial adjustments on the Simon task. Neuroimage 33:399-405.

Kerns JG, Cohen JD, MacDonald AW 3rd, Cho RY, Stenger VA, Carter CS
(2004) Anterior cingulate conflict monitoring and adjustments in control. Science 303:1023-1026.

Konishi S, Kawazu M, Uchida I, Kikyo H, Asakura I, Miyashita Y (1999) Contribution of working memory to transient activation in human inferior prefrontal cortex during performance of the Wisconsin Card Sorting Test. Cereb Cortex 9:745-753.

Logan GD, Zbrodoff NJ (1979) When it helps to be misled: facilitative effects of increasing the frequency of conflicting stimuli in a Stroop-like task. Mem Cognit 7:166-174.

Luks TL, Simpson GV, Dale CL, Hough MG (2007) Preparatory allocation of attention and adjustments in conflict processing. Neuroimage 35:949-958.

MacDonald AW 3rd, Cohen JD, Stenger VA, Carter CS (2000) Dissociating the role of the dorsolateral prefrontal and anterior cingulate cortex in cognitive control. Science 288:1835-1838.

Mansouri FA, Buckley MJ, Tanaka K (2007) Mnemonic function of the dorsolateral prefrontal cortex in conflict-induced behavioral adjustment. Science 318:987-990.

Mansouri FA, Tanaka K, Buckley MJ (2009) Conflict-induced behavioural adjustment: a clue to the executive functions of the prefrontal cortex. Nat Rev Neurosci 10:141-152.

Matsumoto M, Matsumoto K, Abe H, Tanaka K (2007) Medial prefrontal cell activity signaling prediction errors of action values. Nat Neurosci 10:647-656.

Mayr U, Awh E, Laurey P (2003) Conflict adaptation effects in the absence of executive control. Nat Neurosci 6:450-452.

Miller EK, Cohen JD (2001) An integrative theory of prefrontal cortex function. Annu Rev Neurosci 24:167-202.

Nachev P, Rees G, Parton A, Kennard C, Husain M (2005) Volition and conflict in human medial frontal cortex. Curr Biol 15:122-128.

Northoff G, Qin P, Nakao T (2010) Rest-stimulus interaction in the brain: a review. Trends Neurosci 33:277-284.

Petrides M, Pandya DN (2007) Efferent association pathways from the rostral prefrontal cortex in the macaque monkey. J Neurosci 27:11573-11586.

Reynolds JR, West R, Braver T (2009) Distinct neural circuits support transient and sustained processes in prospective memory and working memory. Cereb Cortex 19:1208-1221.

Rissman J, Gazzaley A, D'Esposito M (2004) Measuring functional connectivity during distinct stages of a cognitive task. Neuroimage 23:752-763.

Sakai K, Passingham RE (2003) Prefrontal interactions reflect future task operations. Nat Neurosci 6:75-81.

Simon JR, Rudell AP (1967) Auditory S-R compatibility: the effect of an irrelevant cue on information processing. J Appl Psychol 51:300-304.

Sohn MH, Albert MV, Jung K, Carter CS, Anderson JR (2007) Anticipation of conflict monitoring in the anterior cingulate cortex and the prefrontal cortex. Proc Natl Acad Sci U S A 104:10330-10334.

Song XY, Lee SY (2006) Model comparison of generalized linear mixed models. Stat Med 25:1685-1698.

Soon CS, Brass M, Heinze HJ, Haynes JD (2008) Unconscious determinants of free decisions in the human brain. Nat Neurosci 11:543-545.

Stürmer B, Leuthold H, Soetens E, Schröter H, Sommer W (2002) Control over location-based response activation in the Simon task: behavioral and electrophysiological evidence. J Exp Psychol Hum Percept Perform 28:1345-1363.

Wager TD, van Ast VA, Hughes BL, Davidson ML, Lindquist MA, Ochsner KN (2009) Brain mediators of cardiovascular responses to social threat, part II: Prefrontal-subcortical pathways and relationship with anxiety. Neuroimage 47:836-851.

Zanto TP, Rubens MT, Thangavel A, Gazzaley A (2011) Causal role of the prefrontal cortex in top-down modulation of visual processing and working memory. Nat Neurosci 14:656-661. 